\title{
Sazonalidade e Correlação entre Emissões de Gases Estufa e Variáveis Ambientais em Áreas Florestais Distintas na Amazônia
}

\author{
Aledilson Oliveira dos Reis', Gabriel Brito Costa ${ }^{1}$, Mayara Barbosa Lima1, Marcelo de Almeida Heidemann², \\ Duany Thainara Corrêa da Silva ${ }^{1}$, Ana Caroline da Silva Macambira \\ Letícia Victória dos Santos Matias ${ }^{1} \&$ Natan Barbosa Almada ${ }^{1}$
}

Recebido em 15/09/2020 - Aceito em 27/05/2021

\footnotetext{
1 Universidade Federal do Oeste do Pará/UFOPA, Brasil. <oliverflorestal@hotmail.com, gabrielbritocosta@gmail.com, mayarabarbosaliima@ gmail.com, duanythaynara@gmail.com, karoline_macambira@hotmail.com, lleticia005@gmail.com, natanalmada@gmail.com>.

2 Instituto Federal do Pará/IFPA, Castanhal/PA, Brasil. <marceloaheidemann@hotmail.com>.
}

\begin{abstract}
RESUMO - As especificidades dos padrões sazonais de $\mathrm{CO}_{2}$ e $\mathrm{H}_{2} \mathrm{O}$ atmosférico e suas relações com variáveis meteorológicas em distintas áreas de floresta na Amazônia são um conhecimento extremamente necessário para se construir as bases de protocolos de mitigação às mudanças climáticas, dada a importância da Amazônia na regulação do clima regional e global. Com base nisso, este estudo se propõe a comparar a interação Biosfera-Atmosfera em duas fisionomias distintas de floresta: A FLONA do Tapajós (K67), no Pará, e o Sítio Javaés, localizado no Parque Estadual do Cantão (Ilha do Bananal, Tocantins). Foram analisados os diferentes regimes hídricos, médias horárias e mensais de temperatura e umidade relativa do ar, fluxo de emissão de $\mathrm{CO}_{2}$ pela vegetação e médias horárias e sazonais de concentração de $\mathrm{H}_{2} \mathrm{O}$ e $\mathrm{CO}_{2}$, bem como os padrões horários e sazonais do fluxo turbulento de $\mathrm{CO}_{2}$. Os resultados indicam que o balanço de carbono nos sítios possui grande diferença, onde o sítio do K67 se mostra em equilibbrio em relação as emissões para a atmosfera, podendo atuar como pequena fonte ou sumidouro de $\mathrm{CO}_{2}$ dependendo dos controles energéticos e pluviais do local, enquanto o sítio do Bananal indica um considerável sumidor de $\mathrm{CO}_{2}$ ao longo do ano, tendo suas interações com a atmosfera controlados muito mais pelo ciclo de inundação do sítio.
\end{abstract}

Palavras-chave: Balanço de carbono; fluxos turbulentos; micrometeorologia.

\section{Seasonality and Correlation Between Greenhouse Gas Emissions and Environmental Variables in Different Forest Areas on Amazon}

\begin{abstract}
The specificities of atmospheric $\mathrm{CO}_{2}$ and $\mathrm{H}_{2} \mathrm{O}$ seasonal patterns and their relationship with meteorological variables in different forest areas on Amazon are extremely necessary knowledge to be construe the bases of climate change mitigation protocols, given the importance of the Amazon in the regional and global climate regulation. Based on this, this study proposes to compare the Biosphere-Atmosphere interaction in two different forest physiognomies: FLONA do Tapajós (K67), in Pará, and Javaés site, located in Cantão State Park (Ilha do Bananal, Tocantins). The different water patterns, hourly and monthly averages of temperature and relative air humidity, $\mathrm{CO}_{2}$ emission fluxes through vegetation and hourly and seasonal averages of $\mathrm{H}_{2} \mathrm{O}$ and $\mathrm{CO}_{2}$ concentration were analyzed, as well as hourly and seasonal patterns of turbulent $\mathrm{CO}_{2}$ flux. The results indicate that the carbon balance in the sites has stronger difference, where the $\mathrm{K} 67$ site is neutral in relation to emissions to the atmosphere, and can act as a small source or sink of $\mathrm{CO}_{2}$ depending on the energy and rainfall controls on location, while the Bananal site indicates a considerable $\mathrm{CO}_{2}$ sink throughout the year, having its interactions with the atmosphere controlled more by the site's flood cycle.
\end{abstract}

Keywords: Carbon balance; greenhouse gases; micrometeorology.

\section{Estacionalidad y Correlación entre las Emisiones de Gases de Efecto Invernadero y las Variables Ambientales en Diferentes Áreas Forestales de la Amazonía}

RESUMEN - Las especificidades de los patrones estacionales de $\mathrm{CO}_{2}$ y $\mathrm{H}_{2} \mathrm{O}$ atmosférico y su relación con variables meteorológicas en diferentes áreas forestales de la Amazonía son conocimientos 
sumamente necesarios para construir las bases de los protocolos de mitigación del cambio climático, dada la importancia de la Amazonía en la regulación climática regional y global. Con base en esto, este estudio propone comparar la interacción Biosfera-Atmósfera en dos fisonomías forestales diferentes: FLONA do Tapajós (K67), en Pará, y sitio Javaés, ubicado en el Parque Estatal Cantão (Ilha do Bananal, Tocantins). Se analizaron los diferentes patrones hídricos, promedios horarios y mensuales de temperatura y humedad relativa del aire, flujos de emisión de $\mathrm{CO}_{2}$ a través de la vegetación y promedios horarios y estacionales de concentración de $\mathrm{H}_{2} \mathrm{O}$ y $\mathrm{CO}_{2}$, así como patrones horarios y estacionales de flujo turbulento de $\mathrm{CO} 2$. Los resultados indican que el balance de carbono en los sitios tiene una diferencia más fuerte, donde el sitio K67 es neutral en relación a las emisiones a la atmósfera y puede actuar como una pequeña fuente o sumidero de $\mathrm{CO}_{2}$ dependiendo de los controles de energía y lluvia en el lugar, mientras El sitio Bananal indica un sumidero de $\mathrm{CO}_{2}$ considerable durante todo el año, teniendo sus interacciones con la atmósfera controladas más por el ciclo de inundaciones del sitio.

Palabras clave: Balance de carbono; flujos turbulentos; micrometeorología.

\section{Introdução}

Regiões de floresta tropical como a Amazônia influenciam diretamente na convecção atmosférica e podem afetar outras regiões. Dessa forma, essas regiões são biomas importantes no sistema climático da Terra (Nobre et al., 1991). Estudos de modelagem apontam que as mudanças ou perdas da vegetação amazônica afetam, entre muitos fatores ambientais, principalmente o aumento da temperatura (Galbraith et al., 2010), sendo que é preciso mensurar também a variabilidade dos compostos de carbono produzidos pela vegetação, que influenciam a fotoquímica local, regional e global, exercendo um potencial impacto no clima (Alves et al., 2016). Compostos orgânicos voláteis (COVs) emitidos pela floresta desempenham um papel importante nas relações ecológicas, no ciclo do carbono e na ligação biosfera-atmosfera (Kessler \& Baldwin, 2001; Greene \& Gordon, 2003; Goldstein \& Galbally, 2007; Schmelz et al., 2009), podendo assim afirmar-se que o conhecimento sobre suas emissões se faz tão importante quanto entender o ciclo do carbono regional, dado a correlação entre estes. A dinâmica do carbono $e$ de controles biofísicos da evapotranspiração em ecossistemas tropicais brasileiros e em outras partes do globo tem recebido atenção também pela necessidade de se compreender os efeitos da mudança de uso da terra nos balanços e ciclos biogeoquímicos regionais e globais (Laipelt et al., 2020) e obviamente conhecer a contribuição do bioma Amazônico para o controle climático regional e global, o que envolve o entendimento do regime de precipitação e evapotranspiração, da produtividade vegetal e da troca de gases estufa com a atmosfera (Davidson et al., 2012).
Na Amazônia, apesar de esforços globais em se realizar uma gama cada vez maior de pesquisa, ainda se possui muitas incertezas sobre padrões de emissão de gases estufa e demais compostos para a atmosfera, dado o fato de a Amazônia ser um complexo mosaico de fisionomias com suas particularidades que muitas vezes não se pode extrapolar para uma grande área, necessitando caracterização in situ.

Dado o exposto, fica clara a necessidade de se ampliar a abrangência de estudos sobre o ciclo do carbono em áreas de floresta, sendo o objetivo deste trabalho mostrar as peculiaridades dos padrões sazonais de gases estufa $\left(\mathrm{CO}_{2} e\right.$ $\mathrm{H}_{2} \mathrm{O}$ ), focando suas correlações com variáveis meteorológicas em distintas áreas de floresta na Amazônia: A Flona do Tapajós (Sítio K67/PA), e Parque Estadual do Cantão (Sítio Javaés - Ilha do Bananal/TO), tendo por hipótese que os diferentes regimes hídricos de ambos os sítios possam ter influência direta nos padrões de $\mathrm{CO}_{2}$ locais.

\section{Material e Métodos Dados e localização da área de estudo}

Os dados do projeto LBA utilizados são de domínio público e estão disponíveis em: http:// web.archive.org/web/20150214161815/http:// daac.ornl.gov/LBA/lba.shtml. Foram usados dados coletados de torres de bases micrometeorológicas situadas em dois distintos sítios experimentais, sendo um deles localizados no estado do Pará (Sítio K67, Belterra/PA) e outro no estado do Tocantins (Sítio Javaés, Pium/TO). De acordo com a classificação proposta por Pires \& Prance de (1985) os mais importantes tipos de vegetação amazônica podem ser divididos em grupos 
distintos de tipo de relevo, entretanto neste trabalho levaremos em consideração apenas a classificação sobre as florestas densas (florestas tropicais úmidas ou matas primárias) que podem ser subdivididas em duas grandes categorias: florestas de terra Firme que ocupam maior parte da área total da Amazônia (como o Sítio K67), localizadas em terras mais altas e as florestas inundáveis (como o Sítio Javaés) que representam de 5 a $10 \%$ da bacia Amazônica, estão geralmente situadas nas áreas ao longo dos rios. As áreas selecionadas para estudo estão localizadas na Amazônia legal Brasileira (Figura 1), e dentro do domínio da região Norte do Brasil.

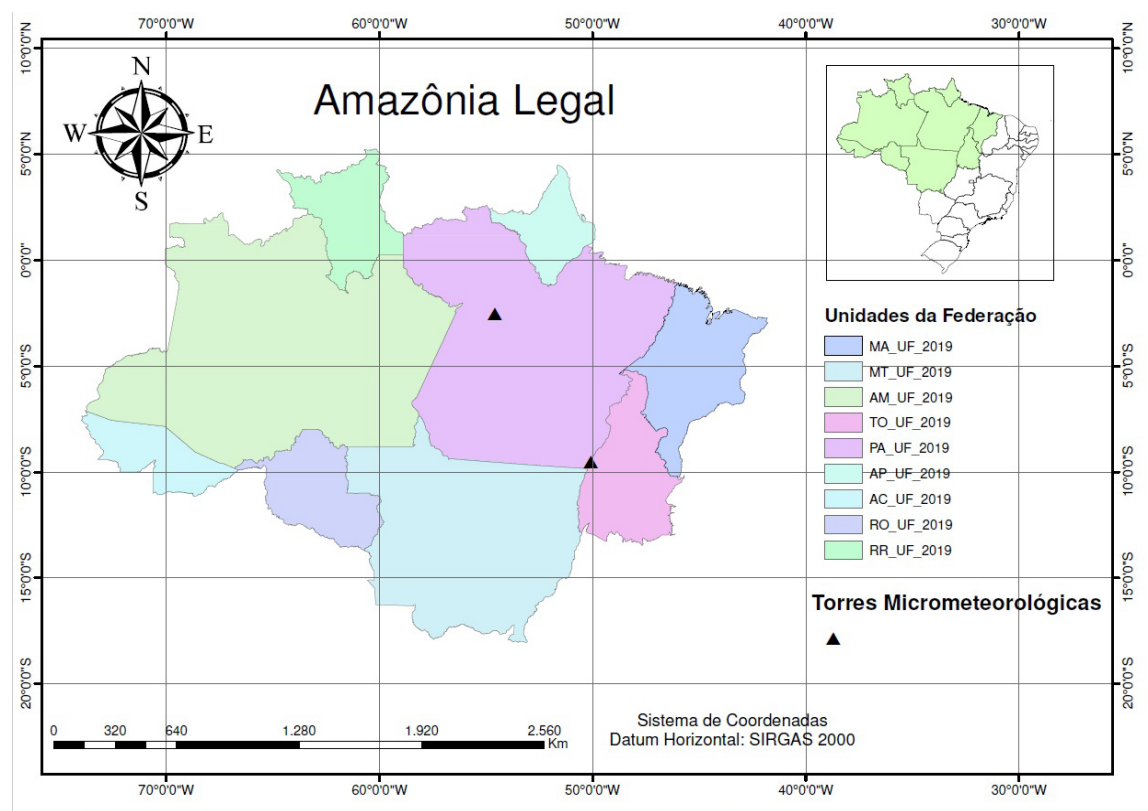

Figura 1 - Localização das torres experimentais nas áreas de estudo no sítio K67/PA e Javaés/TO.

\section{Sítio Javaés - Ilha do Bananal}

O sítio experimental Javaés se situa na Ilha do bananal, de planície de inundação localizada no Parque Estadual do Cantão, distando $260 \mathrm{~km}$ a oeste de Palmas, capital do Tocantins, Brasil. As medições foram feitas em uma torre micrometeorológica instrumentada para a medida de $\mathrm{H}_{2} \mathrm{O}$, energia e $\mathrm{CO}_{2}$, além de variáveis meteorológicas. De acordo com Borma et al. (2009), a torre está situada a $2 \mathrm{~km}$ a leste do Rio Araguaia, nas coordenadas $9^{\circ} 49^{\prime} 27,9 \mathrm{~S}, 50^{\circ}$ 08' 92,8 " W, a $120 \mathrm{~m}$ de altitude, cerca de $1 \mathrm{~km}$ a leste do rio Javaézinho, na fronteira norte da Ilha do Bananal.

A torre micrometeorológica do LBA (Figura 1A) dispunha de sensores instalados acima do dossel vegetativo, em uma região da planície do Araguaia caracterizada por paisagem com aspectos do bioma Cerrado e Amazônico, inclusa em três unidades de conservação, sendo o Parque Nacional do Araguaia, o Parque Estadual do Cantão (PEC) e a Área de Proteção Ambiental do Cantão (SEPLAN, 2004). A Ilha do Bananal abrange uma área de $21.000 \mathrm{~km}^{2}(80 \mathrm{x} 260 \mathrm{~km})$ e é a maior ilha fluvial do mundo, coberta na maior parte por savanas e pastagens, em inundações sazonais geralmente de fevereiro a junho (Borma et al. 2009). O clima regional é quente e sazonalmente úmido, com médias de precipitação anual de $\sim 1466 \mathrm{~mm}$ ano $^{-1}$, aproximadamente $90 \%$ da chuva anual na estação chuvosa entre outubro e abril (Borma et al., 2009; Rocha et al., 2009; Oliveira, 2006) e, portanto, típico do bioma Cerrado no Centro-Oeste do Brasil. As médias mensais de temperatura do ar variam de $25,1^{\circ} \mathrm{C}$ em janeiro a $26,4^{\circ} \mathrm{C}$ em setembro, com a estação seca bem definida entre maio e setembro.

A área de fetch das medidas de fluxos turbulentos da torre cobre três tipos de fisionomia que tendem a acompanhar o nível topográfico: na parte mais alta o Cerradão e florestas semideciduais, na parte intermediária e marcadamente a leste da torre o cerrado, e nas partes mais baixas onde o nível do aquífero é muito raso, há pequenas áreas 
de campo limpo e lagoas isoladas. O desnível dessas áreas é pequeno (Borma et al., 2009). A duração das inundações (início e final, em dias) nos anos em estudo foram: 2004: 30/01-11/06; 2005: 14/02-03/06; 2006: 11/12/05-17/06/2006. A torre de $43 \mathrm{~m}$ fazia medições temperatura do ar, umidade relativa do ar, precipitação pluviométrica e saldo de radiação a uma altura de $40 \mathrm{~m}$, enquanto o sistema de Eddy Covariance com os analisadores de gás e anemômetro sônico estava localizado a $43 \mathrm{~m}$ de altura, sendo que na Tabela 1 há a descrição dos sensores do sítio.
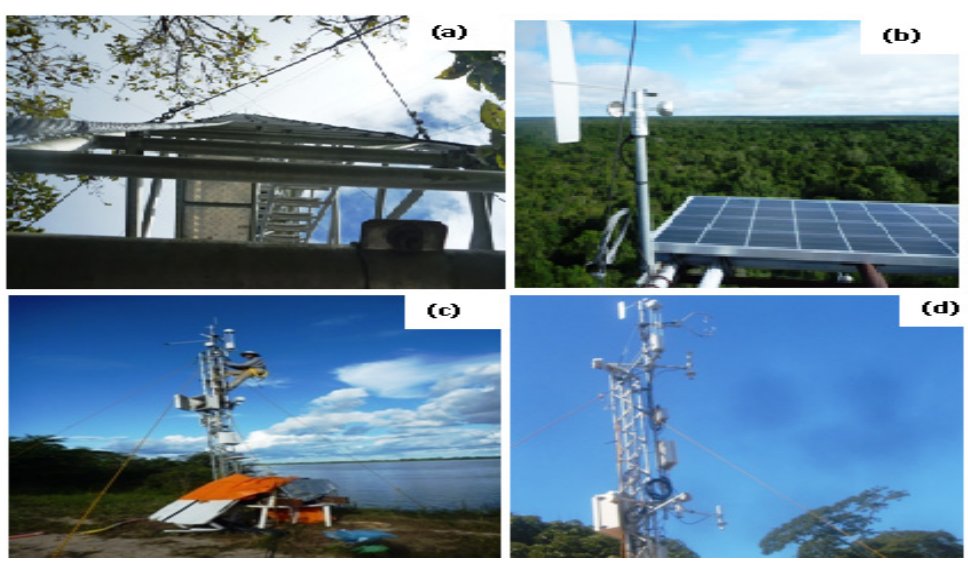

Figura 1A - Vista de base da torre micrometeorológica do LBA no Parque Estadual do Cantão/TO.

Figura 1B - Visão do topo da torre micrometeorológica do LBA, com anemômetro e painéis solares.

Fonte: Costa (2015).

Tabela 1 - Equipamentos disponíveis na torre de 43m em área de Cerrado.

\begin{tabular}{|c|c|}
\hline Medidas/taxa de amostragem/período das médias & Instrumento \\
\hline \multicolumn{2}{|l|}{ Fluxos à superfície } \\
\hline $\begin{array}{l}\text { Eddy covariance: Fluxos turbulentos de calor latente }\left(\mathrm{W} \mathrm{m}^{-2}\right) \text {, } \\
\text { calor sensível }\left(\mathrm{W} \mathrm{m}^{-2}\right) \text { e } \mathrm{CO}_{2}\left(\mathrm{mmol} \mathrm{m}^{-2} \mathrm{~s}^{-1}\right) / 10 \mathrm{~Hz} / 30 \text { minutos e } \\
\text { concentrações de } \mathrm{CO}_{2}(\mathrm{ppm}) \text { e } \mathrm{H}_{2} \mathrm{O}\left(\mathrm{mmol} \mathrm{mol}^{-1}\right)\end{array}$ & $\begin{array}{l}1 \text { Anemômetro sônico Campbell -CSAT3; } 1 \\
\text { Analisador de gás por infravermelho Licor Li-7500 }\end{array}$ \\
\hline \multicolumn{2}{|l|}{ Fluxos de radiação } \\
\hline Saldo de radiação $\left(\mathrm{Wm}^{-2}\right) / 1$ minuto/30 minutos & 1 Saldo radiômetro Kipp-Zonen NR Lite \\
\hline \multicolumn{2}{|l|}{ Variáveis climatológicas } \\
\hline $\begin{array}{l}\text { Temperatura }\left({ }^{\circ} \mathrm{C}\right) \text { e umidade relativa do ar }(\%) / 1 \text { minuto/30 } \\
\text { minutos }\end{array}$ & 1 termo-higrômetro VAISALA HMP45C \\
\hline
\end{tabular}

Fonte: Costa, 2015.

\section{Sítio Experimental K67}

A Floresta Nacional do Tapajós está em um domínio de aproximadamente 560.000ha, localizada nas proximidades da foz do rio Tapajós, compreendendo áreas dos municípios de Belterra, Rurópolis, Placas e Aveiro, todos municípios do estado do Pará. O clima da região é do tipo tropical úmido de monção, com temperatura média anual de $25,5^{\circ} \mathrm{C}$ (Machado, 2012). De acordo com D’Amelio (2006) a estação chuvosa está compreendida entre os meses de janeiro a julho, quando a precipitação é acima de $50 \mathrm{~mm}$, $e$ a estação seca ocorre entre os meses de agosto a dezembro, sendo mais acentuada nos meses de agosto a outubro. O relevo é plano ou dissecado, as superfícies de aplainamento são inundadas periodicamente por ocasião das chuvas (Ibama, 2004).

De acordo com Veloso (1991), a vegetação é classificada como Floresta Ombrófila Densa 
das Terras Baixas, caracterizando-se pela dominância de indivíduos arbóreos de grande porte e pela abundância de lianas lenhosas, palmeiras e epífitas. A torre micrometeorológica K67 (02 $51^{\circ}$ S, 54 58' W) situada na Floresta
Primária está localizada a cerca de $67 \mathrm{~km}$ da cidade de Santarém/PA, fora instalada $\mathrm{a}$, aproximadamente, $6 \mathrm{~km}$ de distância, em linha reta da rodovia (Figura 2 ).
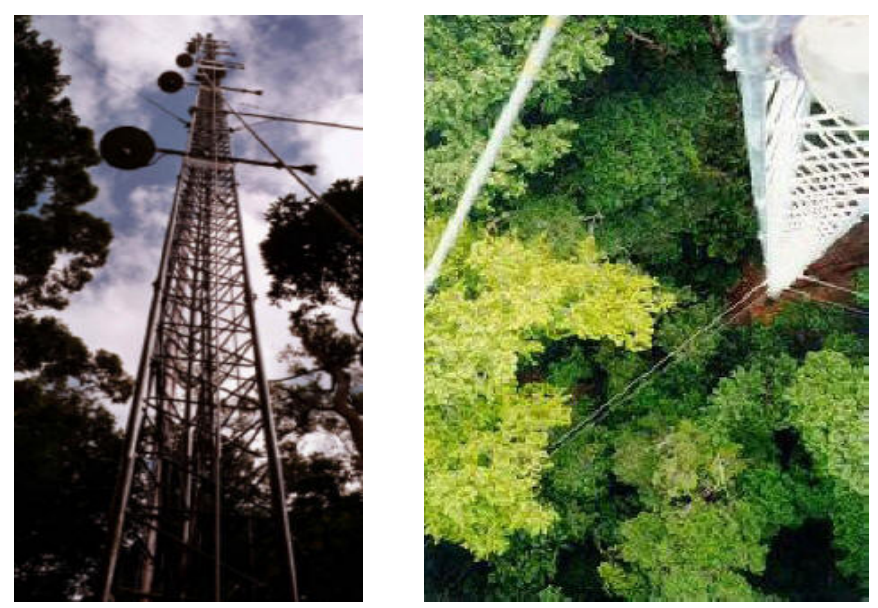

Figura 2 - Torre K67 do Programa LBA. À direita, vista da torre K67 com 64m de altura, e à esquerda sub-bosque da floresta vista de cima. Fonte: Hutyra et al. (2007).

Os dados utilizados foram medidos no âmbito do Programa LBA (Programa de Grande Escala da Biosfera-Atmosfera na Amazônia), o conjunto de dados do LBA compreende medidas horárias de diversas variáveis ambientais. Esses dados foram medidos pelos sensores da torre micrometeorológica instaladas $e$ instrumentada com o objetivo principal de verificar os processos físicos, biofísicos e biológicos entre biosfera atmosfera (Saleska et al., 2003; Hutyra et al., 2007; Rocha et al., 2009), sendo que na Tabela 2 há a descrição dos sensores do sítio.

Tabela 2 - Instrumentação de campo na Floresta (km67).

\begin{tabular}{|c|c|c|c|}
\hline Componentes & Modelo & Nível vertical (m) & Variável medida* \\
\hline Anemômetro sônico 3D & CSAT3 & 57,79 & $\mathrm{U}_{\mathrm{x}}, \mathrm{U}_{\mathrm{y}}, \mathrm{U}_{\mathrm{z}}$ \\
\hline Analisador de gás por infravermelho & LiCor LI-6262 & $\begin{array}{l}62,24 ; 50,05 ; 39,41 ; 28,71 \\
19,57 ; 10,42 ; 3,05 ; 0,91\end{array}$ & $\mathrm{H}_{2} \mathrm{O}, \mathrm{CO}_{2}$ \\
\hline $\begin{array}{l}\text { Sensor de temperatura e umidade } \\
\text { relativa do ar }\end{array}$ & CS500 & 2 & $\mathrm{~T}_{\mathrm{ar}}, \mathrm{UR}$ \\
\hline Saldo-radiômetro & RebsQ7,1 & 64,1 & $Q^{*}$ \\
\hline Velocidade do vento & $\begin{array}{l}\text { Spinning cup anemometer, } \\
\text { MetOne010C }\end{array}$ & 64,$1 ; 52,3 ; 8,2$ e 30,7 & WS \\
\hline Direção do vento & MetOne020C & 64,1 & WD \\
\hline Pluviômetro & Texas Eletronics 076B-4 & 42,6 & Precipitação \\
\hline
\end{tabular}

Fonte: Machado (2012).

\section{Processamento de dados}

O processamento de dados e análises estatísticas foi feito com uma linguagem $e$ ambiente de desenvolvimento integrado $\mathrm{R}, \mathrm{R}$ Core Team (2020). Os dados de alta frequência geram fluxos de 30 minutos calculados usando o software de código aberto EddyPro ${ }^{\circledR}$ Eddy Covariance 
Processing, versão 6.2.2 (LI-COR Biosciences, Lincoln, NE, EUA). Os dados brutos de alta frequência utiliza médias de fluxos turbulentos $\mathrm{H}$ e LE, aplicação de correções envolvendo: spike filtering; rotação dos eixos do sônico (Aubinet et al., 1999; Wilczak et al., 2001); rotação de coordenadas usando o método de ajuste planar descrito em Wilczak et al. (2001), correção de temperatura virtual do sônico (Kaimal \& Gaynor, 1991), bem como a correção de resposta de frequência incorporada derivada de Moore (1986) e Massman (2000) para melhoramento dos dados de fluxo. Dados espúrios foram detectados conforme método de Papale et al. (2006), e o preenchimento de falhas de dados diurnos de $\mathrm{CO}_{2}$ foi feito usando curvas de luz com a radiação PAR, enquanto os preenchimentos de fluxos fracos devido baixa turbulência foram preenchidos com médias de dados válidos, conforme as metodologias descritas por Hutyra et al. (2007) e Costa (2015). A técnica de Eddy covariance na estimativa de fluxos de energia e $\mathrm{CO}_{2}$ é amplamente empregadas na literatura, tanto para estudos em área de floresta (Araújo et al., 2002; Rocha et al., 2009; Rocha et al., 2002; Hutyra et al., 2007; Campos et al., 2019), agroecossistemas (Veeck et al., 2018), assim como para estimativas em área de pastagem (Acosta et al., 2020; Alves, 2015) ou outras superfícies peculiares, como reservatórios (Vale et al., 2016), sendo no caso deste artigo, o fluxo líquido do ecossistema é considerado como proxy do fluxo turbulento medido pelo Eddy Covariance (sem dados de armazenamento), e a respiração trata dos dados noturnos do mesmo dado.

\section{Resultados e Discussão}

\section{Elementos meteorológicos}

As condições climáticas médias sazonais $e$ horárias dos elementos meteorológicos (Figura 3) temperatura do ar, umidade relativa do ar e precipitação normalizada para o período de estudo de janeiro de 2004 a janeiro de 2006 são mostradas a seguir.
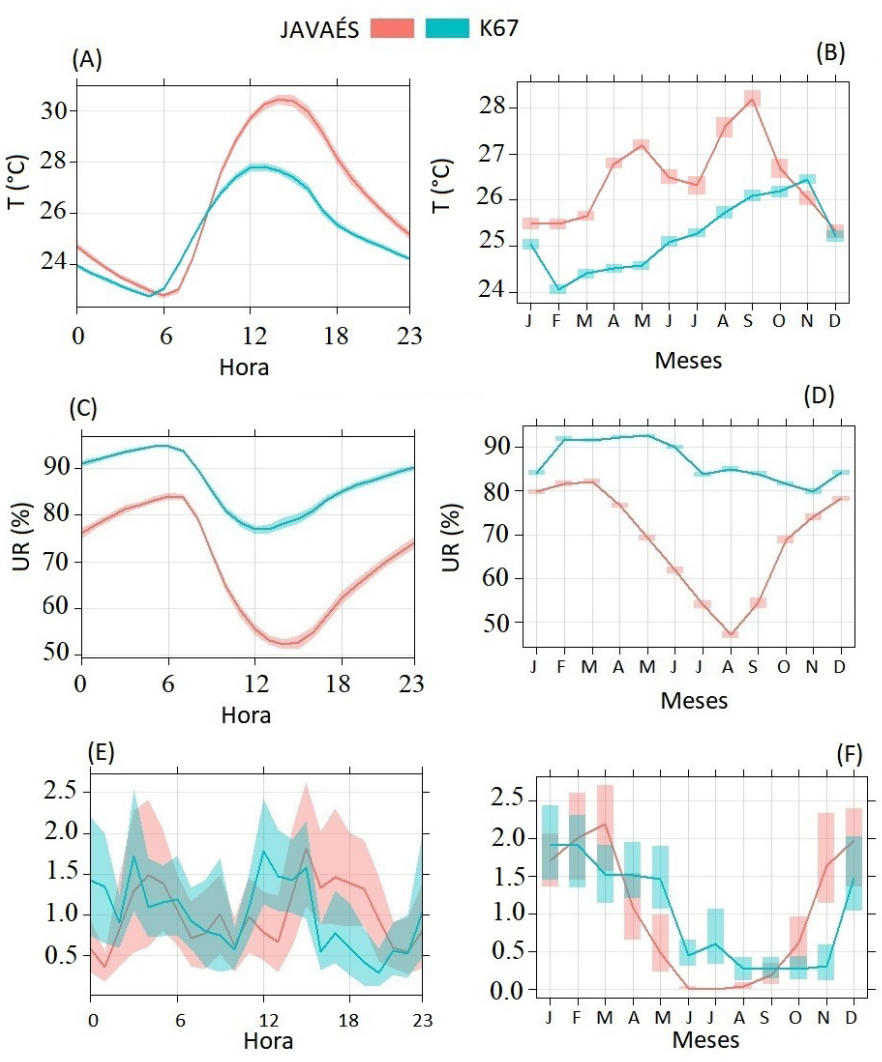

Figura 3 - Perfil médio horário e variação sazonal de temperatura do ar (painéis A e B), umidade relativa do ar (painéis $\mathrm{C}$ e $\mathrm{D}$ ) e precipitação normalizada (painéis $\mathrm{E}$ e $\mathrm{F}$ ) para os sítios em estudo. As áreas hachuradas representam o intervalo de confiança (95\%) dos dados. 
A temperatura do ar (Figura 3A) é mais elevada no Sítio Javaés, onde atinge o máximo no mês de setembro (Figura 3B), enquanto no Sítio K67 o máximo é atingido no mês de novembro. A diferença nos padrões térmicos dos sítios fica evidente com o fato dos máximos de temperatura do ar do Sítio K67 ter valores próximos aos mínimos de temperatura do Sítio Javaés, pouco acima dos $26^{\circ} \mathrm{C}$. Apesar de ter uma estação seca com precipitação bem abaixo dos meses da estação úmida, a umidade relativa do Sítio K67 é maior (Figura 3C) e elevada o ano todo (acima de $80 \%$, Figura 3D), enquanto no Sítio Javaés a umidade relativa decai acentuadamente no período menos chuvoso, até atingir seu mínimo (abaixo de 50\%) no mês de agosto (Figura 3D).

Os sítios possuem diferenças nos padrões de precipitação, onde os meses mais chuvosos se concentram entre dezembro e abril, com uma estação seca média de 5 a 6 meses de duração (meses com precipitação inferior a $100 \mathrm{~mm}$ acumulados), sendo que a retomada das chuvas após os meses de estiagem no Sítio Javaés ocorreu a partir de setembro, enquanto no Sítio K67 ocorreu a partir de novembro. Estes padrões estão dentro dos reportados quanto a precipitação e duração da estação chuvosa na bacia Amazônica (Saleska et al., 2003). A precipitação horária (Figura 3E) e mensal (Figura 3F) está normalizada, que consiste em dividir os valores observados pela média dos períodos, de modo a ajudar a comparar a forma das tendências diurnas e mensais para escalas diferentes. Existe tendência para a precipitação ocorrer no período da tarde (1200-1500 no Sítio K67, 1500-1800 no Sítio Javaés, hora local) provavelmente devido à atividade convectiva estimulada pelo aquecimento da superfície, como já reportado em outros estudos na área do sítio do K67 (Hutyra et al., 2007), e agora também verificado no Sítio Javaés.

O Sítio K67 se mostrou mais úmido, até mesmo por sua característica de floresta tropical úmida (Rocha et al., 2009) onde a precipitação média pode atingir próximo de $2000 \mathrm{~mm}$ anuais (Hutyra et al., 2007), enquanto o sítio do Javaés, por estar em uma região de planície inundável sazonal e ecotonal, com fisionomias de cerrado e cerradão (Rocha et al., 2009; Borma et al.,
2009) pode apresentar acumulados anuais de precipitação pouco acima de $1200 \mathrm{~mm}$ anuais em anos mais secos (Costa, 2015), o que justifica os máximos de umidade relativa mensal do Javaés se mostrarem menores que os mínimos de umidade relativa do Sítio K67, da mesma forma no perfil médio horário.

\section{Fluxos de energia}

A variabilidade dos componentes do balanço de energia (Figura 4) fluxo de calor sensível $(\mathrm{H})$, fluxo de calor latente (LE) e saldo de radiação $(\mathrm{Rn})$ para o período de estudo de janeiro de 2004 a janeiro de 2006 é mostrada a seguir.

Devido aos padrões de fisionomias vegetais distintos já comentados entre os sítios, os fluxos de energia refletem estas diferenças fisionômicas $e$ fisiológicas da vegetação. Enquanto no sítio de floresta tropical úmida (K67) os fluxos de calor sensível (Figura 4A e Figura 4B) acompanham a oferta radiativa ao longo do ano (Araújo et al., 2010; Rocha et al., 2009; Hutyra et al., 2007), no sítio inundável do cerrado (Javaés) há uma inversa proporcionalidade entre a energia disponível (Rn máximo) e o fluxo de calor latente (LE mínimo), principalmente com a proximidade do auge da estação seca (setembro), conforme já identificado em outros trabalhos de áreas similares de cerrado (Biúdes et al., 2009) dado que LE é maior que $\mathrm{H}$ tanto na fase seca quanto na fase úmida no Sítio $\mathrm{K} 67$, enquanto que $\mathrm{H}$ ultrapassa LE na fase seca do Sítio Javaés e LE é máximo nos meses em que o sítio está inundado (janeiro a março).

A inundação que ocorre no Sítio Javaés nos meses iniciais do ano, associado com o efeito de rebrota de folhas verdes na estação chuvosa dos cerrados (Rocha et al., 2002) e possível controle estomatal devido o intenso calor (Costa, 2015) seriam os motivos para este padrão diferenciado em relação outros tipos de floresta tropical, uma vez que o grande volume de água no sítio impulsiona o fluxo de calor latente, a rebrota das folhas altera o padrão de albedo e o controle estomatal faz com que o fluxo de calor sensível fique maior que o fluxo de calor latente. 

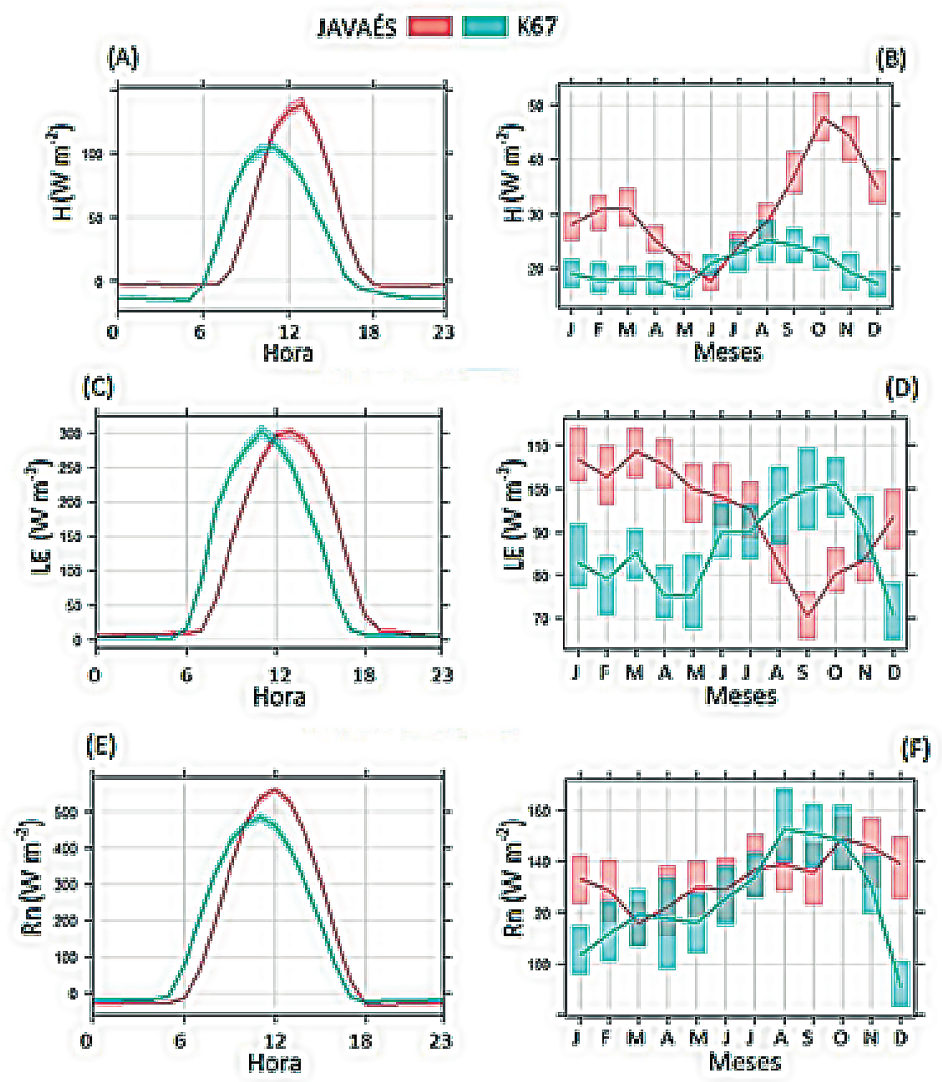

Figura 4 - Perfil médio horário e variação sazonal do fluxo de calor sensível (painéis A e B), fluxo de calor latente (painéis $\mathrm{C}$ e $\mathrm{D}$ ) e saldo de radiação (painéis $\mathrm{E}$ e $\mathrm{F}$ ) para os sítios em estudo. As áreas hachuradas representam o intervalo de confiança $(95 \%)$ dos dados.

\section{Padrões de gases estufa $\left(\mathrm{CO}_{2}\right.$ e $\left.\mathrm{H}_{2} \mathrm{O}\right)$}

A variabilidade das concentrações de $\mathrm{CO}_{2}, \mathrm{H}_{2} \mathrm{O}$ e fluxo turbulento de $\mathrm{CO}_{2}$ (Figura 5) para o período de estudo de janeiro de 2004 a janeiro de 2006 são mostradas a seguir. Devido à falta de medidas de armazenamento de $\mathrm{CO}_{2}$, a produtividade líquida do ecossistema (contabilização para determinar se o ambiente é um sumidouro ou fonte de $\mathrm{CO}_{2}$ para a atmosfera) pode ser tomada como medida proxy do fluxo turbulento medido (Borma et al., 2009; Rocha et al., 2002). Os perfis médios horários de $\mathrm{CO}_{2}$ (Figura 5A) mostram maior variabilidade no sítio do Javaés do que no Sítio K67.

Existe pouca variação ao longo do ano nas concentrações do Sítio K67 (Figura 5B), não ultrapassando $10 \mathrm{pmm}$ entre mínimos e máximos mensais, enquanto o Sítio Javaés tem variação superior a $30 \mathrm{ppm}$ entre os meses, evidenciando as particularidades hídricas dos sítios, onde o Javaés reproduz um forte padrão de savana (Costa, 2015) com uma estação seca que reduz fortemente o fluxo de calor latente, enquanto o Sítio K67 tem padrões característicos de Floresta Tropical úmida (Hutyra et al., 2007). Embora não haja grande variabilidade horária, há grande diferença nas concentrações de $\mathrm{H}_{2} \mathrm{O}$ (Figura 5C), sendo o Sítio $\mathrm{K} 67$ 2,5 vezes mais úmido. As diferenças sazonais de $\mathrm{H}_{2} \mathrm{O}$ (Figura 5D) são pouco mais acentuadas no Sítio Javaés, com pouca variabilidade no Sítio K67. Os padrões de assimilação de $\mathrm{CO}_{2}$ horários (Figura 5E) e mensais (Figura 5F) possuem diferenças, com uma extensão do período de respiração do ecossistema (valores positivos) até às $08 \mathrm{~h}$ locais no Sítio Javaés, enquanto o Sítio K67 já possui padrão de assimilação (valores negativos) desde às $07 \mathrm{~h}$ locais.

A pouca variabilidade nas concentrações de $\mathrm{CO}_{2}$ do Sítio $\mathrm{K} 67$ não ocorre no Sítio Javaés. Isto se deve provavelmente ao efeito de anóxia que a vegetação passa nos meses iniciais do ano com a ocorrência das inundações (Borma et al., 2009; Oliveira, 2006; Costa, 2015) que faz com que as 
(A)
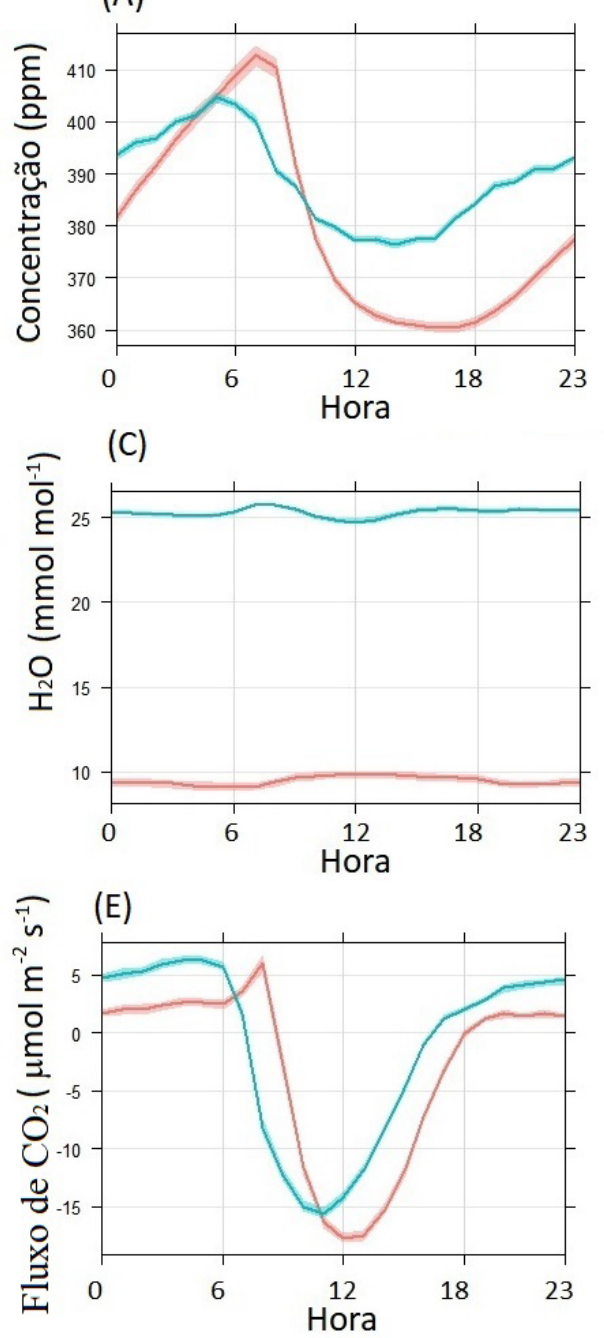

K67

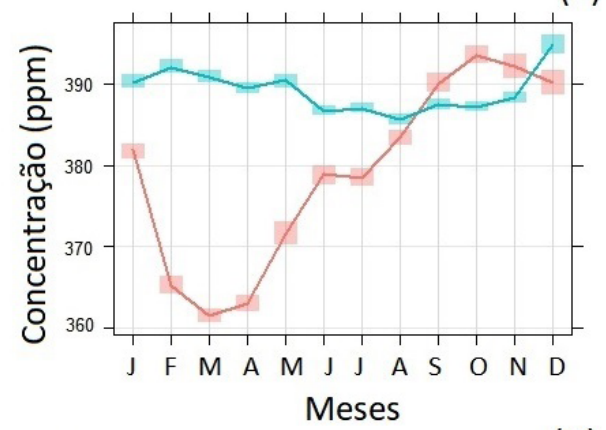

(D)

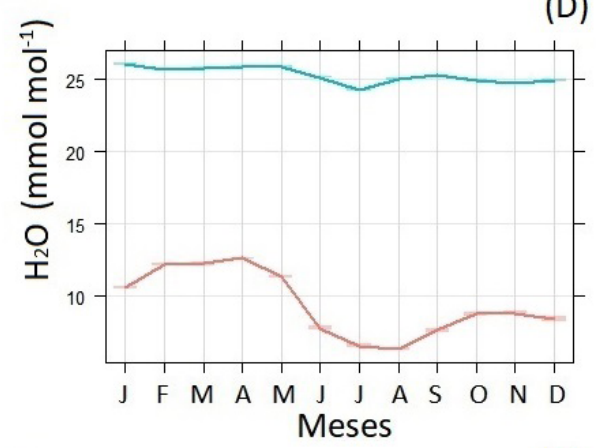

(F)

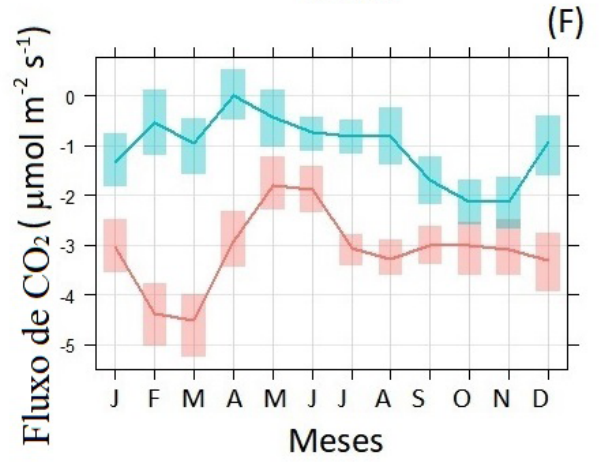

Figura 5 - Perfil médio horário e variação sazonal da concentração de $\mathrm{CO}_{2}$ (painéis $\mathrm{A}$ e B), concentração de $\mathrm{H}_{2} \mathrm{O}$ (painéis $\mathrm{C}$ e D) e fluxo turbulento de $\mathrm{CO}_{2}$ (painéis $\mathrm{E}$ e F) para os sítios em estudo. As áreas hachuradas representam o intervalo de confiança (95\%) dos dados.

emissões diminuam gradativamente até o mês de março. A decomposição de matéria orgânica e retomada da atividade metabólica das plantas após a inundação produz um forte pulso de emissão de $\mathrm{CO}_{2}$ que se estende até o mês outubro. Os altos valores de assimilação (fluxo negativo) mostrados pelo Sítio Javaés (Figura 5F) já foram reportados em outros trabalhos em área de floresta tropical preservada (Araújo et al., 2002) e cerrado (Biúdes et al., 2021; Costa, 2015), sendo explicados por menores valores de respiração noturna e assimilações mais intensas durante o dia. O Sítio K67 quanto o balanço de carbono anual, se aproxima de uma neutralidade (Saleska et al., 2003; Hutyra et al., 2007) onde alguns anos podem apresentar um pequeno sumidouro de $\mathrm{CO}_{2}$ e outros podem representar uma pequena fonte, condicionado as condições de precipitação e disponibilidade energética na região. Já o sítio Javaés possui seus padrões de ciclo do carbono fortemente influenciados pela inundação do sítio e a umidade do solo (Borma et al., 2009; Costa, 2015), motivo pelo qual o período mais quente do ano não é também o com menores fluxos, a exemplo do que acontece no Sítio K67.

\section{Correlações entre as variáveis ambientais}

As correlações (correlação de Pearson) entre as variáveis medidas nos sítios são mostradas a 
seguir. Além das variáveis já analisadas, foi adicionada a velocidade de fricção (u*) que é um parâmetro importante utilizado nas correções de fluxos noturnos e mensura a intensidade turbulenta do local.
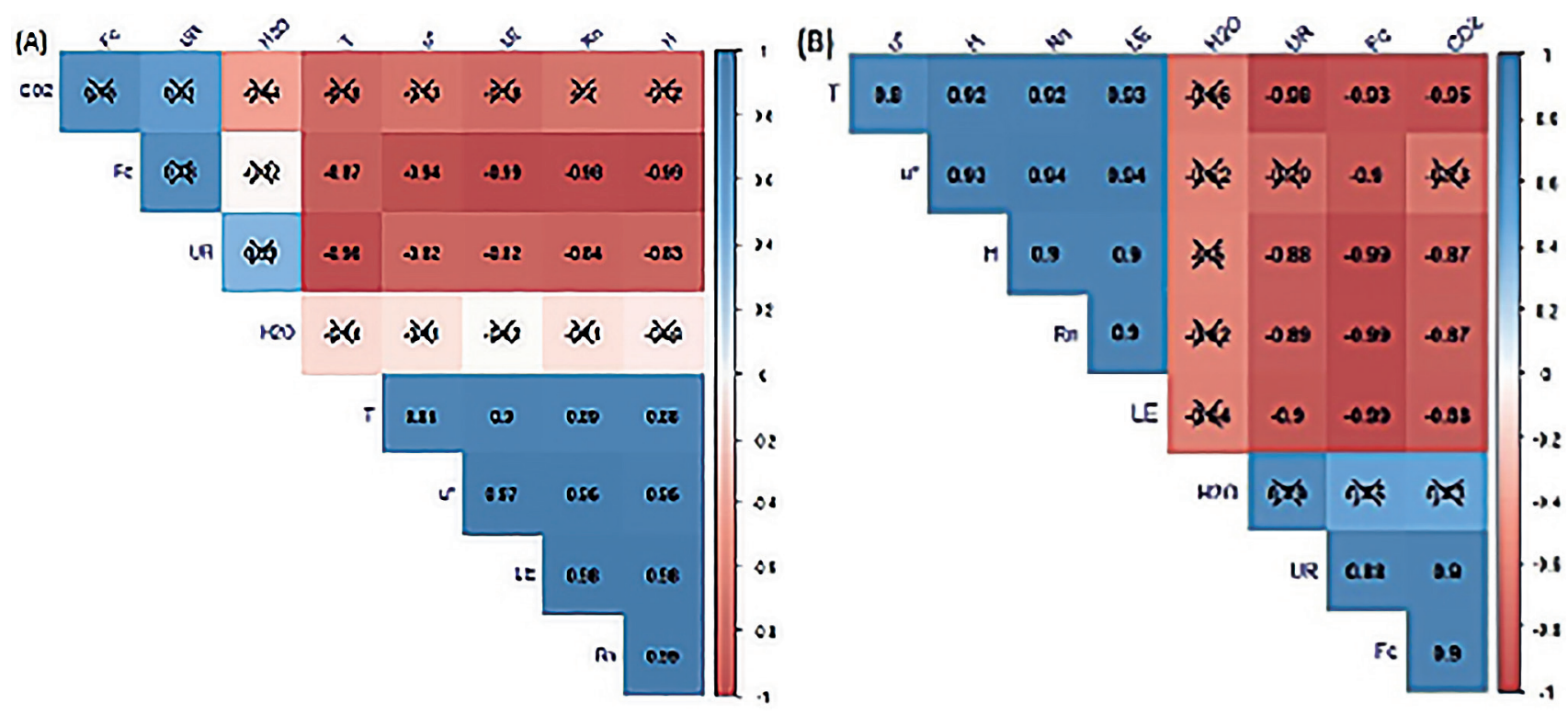

Figura 6 - Heatmap com base na matriz de correlação (teste de correlação de Pearson) com as variáveis medidas no Sítio Javaés (A) e K67 (B): Fc - Fluxo turbulento de $\mathrm{CO}_{2}\left(\mu \mathrm{mol} \mathrm{m} \mathrm{m}^{-2} \mathrm{~s}^{-1}\right), \mathrm{H}$ - fluxo de calor sensível $\left(\mathrm{W} \mathrm{m}^{-2}\right), \mathrm{LE}$ - fluxo de calor latente $\left(\mathrm{W} \mathrm{m}^{-2}\right), \mathrm{Rn}$ - saldo de radiação $\left(\mathrm{W} \mathrm{m}^{-2}\right)$, $\mathrm{UR}$ - umidade relativa do ar $(\%), \mathrm{T}$ - temperatura do ar $\left({ }^{\circ} \mathrm{C}\right)$, $\mathrm{u}^{*}$ - velocidade de friç̧ão $\left(\mathrm{m} \mathrm{s}^{-1}\right), \mathrm{CO}_{2}$ - concentrações de $\mathrm{CO}_{2}$ (ppm) e $\mathrm{H}_{2} \mathrm{O}$-concentrações de $\mathrm{H}_{2} \mathrm{O}\left(\mathrm{mmol} \mathrm{mol}^{-1}\right)$. Cores frias representam correlação positiva e cores quentes representam correlações negativas com base no teste de correlação de Pearson $(p<0,05)$. $\mathrm{O}$ "X" nos coeficientes de correlação indicam correlações não significativas.

Nota-se que os padrões de temperatura do ar são inversamente correlacionados com os fluxos de $\mathrm{CO}_{2}$ em ambos os sítios, mais fortemente correlacionado no sítio do K67 (Fig. 6B). Os fluxos de energia são fortemente correlacionados entre sí em ambos os sítios, sendo que o fluxo turbulento de $\mathrm{CO}_{2}$ possui padrão distinto quanto a correlação com a umidade do ar no Sítio Javaés (não significativo) e K67 (significativo). Isto pode refletir o efeito da inundação do sítio, que é quem mais influencia no controle das trocas de $\mathrm{CO}_{2}$ do que a oferta radiativa (Borma et al., 2009; Costa, 2015). Já o Sítio K67 tende que a limitação de umidade na estação seca gere forte restrição na absorção de carbono pelo dossel, conforme estudos de Botta et al. (2002) e Tian et al. (1998). Os padrões de alta produtividade do Sítio Javaés são defensáveis pela hipótese de que a absorção de $\mathrm{CO}_{2}$ por árvores vivas é superior a emissão por árvores mortas, indicando que o efeito geral da floresta é a absorção (Espirito Santo et al., 2014).

\section{Conclusões}

A sazonalidade dos padrões de $\mathrm{CO}_{2}$ nos sítios analisados se mostrou bastante influenciadas por variações sazonais na pluviosidade e fatores hidrológicos (inundação) em um dos sítios. Os controles radiativos e de pluviosidade podem tornar o Sítio $\mathrm{K} 67$ fonte ou sumidouro de $\mathrm{CO}_{2}$ para a atmosfera, enquanto o Sítio Javaés se mostrou com padrão de sumidouro constante. Os diferentes regimes hídricos, assim com as interações biogeoquímicas que ocorrem com o solo inundado em um dos sítios, são prováveis motivos dos padrões de $\mathrm{CO}_{2}$ distintos entre ambos. O Sítio K67 é muito mais úmido que o Sítio Javaés, $e$ as particularidades fisionômicas e fisiológicas da vegetação fazem com que a oferta radiativa não seja a variável que controla a evapotranspiração no Sítio Javaés, dado que no auge da estação seca há um forte decréscimo do fluxo de calor latente, mesmo com o aumento do saldo de radiação. 
Há forte influência da inundação que ocorre no sítio nos fluxos de energia, sendo o máximo de LE justamente neste período e concomitante com redução nos fluxos turbulentos de $\mathrm{CO}_{2}$, provavelmente por um efeito de anóxia.

\section{Referências}

Acosta R, Veeck GP, Bremm T, Roberti DR \& Moraes OLL. Impacto do uso do filtro - velocidade de fricção - em estimativas anuais de carbono sobre ecossistemas de pastagem natural. Ciência e Natura, 42: e7, 2020.

Alves EM. Fluxos de energia, vapor d'água e $\mathrm{CO}_{2}$ entre a vegetação e a atmosfera no agreste meridional de Pernambuco. Recife: Universidade Federal de Pernambuco. 96p. Tese Doutorado, 2015.

Aubinet $\mathrm{M}$ et al. Estimates of the annual net carbon and water exchange of forests: the EUROFLUX methodology, Adv. Eco. Res., 30: 113-175, 1999.

Alves EG et al. Seasonality of isoprenoid emissions from a primary rainforest in central Amazonia. Atmospheric Chemistry and Physics, 16: 3903-3925, 2016.

Araújo AC et al. Comparative measurements of carbon dioxide fluxes from two nearby towers in a central Amazonian rainforest: The Manaus LBA site, Journal of Geophysical Research, New York, 107(D20): 8090, 2002.

Biudes Ms, Campelo Júnior JH, Nogueira JS \& Sanches L. Estimativa do balanço de energia em cambarazal e pastagem no norte do Pantanal pelo método da razão de Bowen. Revista Brasileira de Meteorologia. São José dos Campos, 24(2): 135-143, 2009.

Biúdes MS et al. Gross primary productivity of Brazilian Savanna (Cerrado) estimated by different remote sensing-based models. Agricultural and Forest Meteorology, 307: 108456, 2021.

Borma LS et al. Atmosphere and hydrological controls of the evapotranspiration over a floodplain forest in the Bananal Island region, Amazonia. Journal of Geophysical Research, New York, 114: 1-12, 2009.

Botta A, Ramankutty N \& Foley JA. Long-term variations of climate and carbon fluxes over the Amazon basin. Geophysical Research Letters, 29: 1319. 2002.

Campos $\mathrm{S}$ et al. Closure and partitioning of the energy balance in a preserved area of Brazilian seasonally dry tropical forest. Agric. For. Meteorol. 271: 398-412. https://doi.org/10.1016/j.agrformet.2019.03.018. 2019.

Costa GB. Fluxos de energia, $\mathrm{CO}_{2}$ e $\mathrm{CH}_{4}$ sobre a floresta em planície de inundação da ilha do Bananal. Tese (Doutorado), Escola Superior de Agricultura "Luiz de Queiroz", 142p. 2015.
Davidson EA et al. The Amazon basin in transition. Nature, London, v. 481: 321-328, 2012.

D'Amelio MTS. Estudo de gases de efeito estufa na Amazônia. 178p. Dissertação (Mestrado) - Instituto de Pesquisas Energética e Nucleares, Programa de Pós-Graduação em Ciências, na área de Tecnologia Nuclear, São Paulo, 2006.

Espirito-Santo FDB et al. Size and frequency of natural forest disturbances and the Amazon forest carbon balance. Nature Communications. New York, 5: 3434 doi: 10.1038/ncomms4434, 2014.

Galbraith D, Levy PE, Sitch S, Huntingford C, Cox P, Williams M \& Meir P. Multiple mechanisms of Amazonian forest biomass losses in three dynamic global vegetation models under climate change. New Phytologist, 187: 647-665, 2010.

Goldstein AH \& Galbally IE. Known and Unexplored Organic Constituents in the Earth's Atmosphere. Environmental Science \& Technology, 41: 1514-1521, 2007.

Greene MJ \& Gordon DM. Cuticular hydrocarbons inform task decisions. Nature, 423: 32-32, 2003.

Hutyra LR, Munger W, Saleska S, Gottlieb E, Daube BC, Dunn AL, Amaral DF, Camargo PB \& Wofsy S. Seasonal controls on the exchange of carbon and water in an Amazonian rain forest. Journal of Geophysical Research, New York, 112: 1-16, 2007.

Kaimal JC \& Gaynor J. Another look at sonic thermometry. Boundary-Layer Meteorol 56: 401-410, 1991.

Kessler A \& Baldwin I. Defensive Function of HerbivoreInduced Plant Volatile Emissions in Nature. Science, 291: 2141-2144, 2001.

Laipelt L, Ruhoff AL, Fleischmann AS, Kayser RHB, Kich EM, Da Rocha HR \& Neale CMU. Assessment of an Automated Calibration of the SEBAL Algorithm to Estimate Dry-Season Surface-Energy Partitioning in a Forest-Savanna Transition in Brazil. Remote Sens. 12: 1108, 2020.

Machado WB. Balanço de energia em áreas de floresta e de um campo agrícola no leste da Amazônia. Dissertação apresentada à Universidade Federal do Oeste do Pará/UFOPA, Santarém, Pará, 2012.

Massman WJ. A simple method for estimating frequency response corrections for eddy covariance systems. Agric. For. Meteorol. 104: 185-198, 2000.

Moore CJ. Frequency response corrections for eddy correlation systems, Bound.-Lay. Meteorol., 37: 17-35, 1986.

Nobre CA, Sellers PJ \& Shukla J. Amazonian deforestation and regional climate change. Journal of Climate. 957-988, 1991. 
Oliveira LS. Fluxos atmosféricos de superfície sobre uma área de ecótono na Ilha do Bananal. 97f. Tese (Doutorado em Meteorologia). Universidade de São Paulo, São Paulo, 2006.

Papale D et al. Towards a standardized processing of Net Ecosystem Exchange measured with eddy covariance technique: algorithms and uncertainty estimation. Biogeoscience 3: 571-583, 2006.

Pires JM \& Prance GT. The vegetation types of the Brazilian Amazonia. In: Prance GT, Lovejoy TE (Eds). Key environments: Amazonia. Pergamon Press, New York, 109-145, 1985.

Rocha HR, Freitas HC, Rosolem R, Juarez RIN, Tannus RN, Ligo MA, Cabral OMR \& Dias MAFS. Medidas de fluxos de $\mathrm{CO}_{2}$ em um Cerrado sensu stricto no sudeste do Brasil. Biota Neotropica, Campinas, 2: 1, 2002.

Rocha HR, Manzi AO, Cabral OM, Miller SD, Goulden ML, Saleska SR \& Coupe NR. Patterns of water and heat flux across a biome gradient from tropical forest to savanna in Brazil. Journal of Geophysical Research, New York, 114: 1-8, 2009.

Saleska SRSR et al. Carbon in Amazon forests: Unexpected seasonal fluxes and disturbance-induced losses. Science,Washington, 302(5650): 1554-1557, 2003.

SEPLAN (Secretaria de Planejamento). Plano de Manejo do Parque Estadual do Cantão. Secretaria de
Planejamento e Meio Ambiente. Governo do Estado do Tocantins, 85p. 2004.

Schmelz EA et al. Phytohormone-based activity mapping of insect herbivore-produced elicitors. Proceedings of the National Academy of Sciences, 106(2), 2009.

Tian H, Melillo JM, Kicklighter DW, McGuire AD, Helfrich JVK, Moore B \& Vorosmarty CJ. Effect of interannual climate variability on carbon storage in Amazonian ecosystems. Nature, 396: 664-667, 1998.

Vale RS et al. Medições por covariância de vórtices turbulentos dos fluxos de calor latente, sensível, momentum e $\mathrm{CO}_{2}$ sobre o reservatório da Usina Hidrelétrica de Curuá-Una/PA. Ciência e Natura, 38: 15, 2016.

Veeck GP, Teichrieb C, Diaz M, Bremm T, Oliveira ME, Roberti DR, Amado TJ, Fiorin JE \& Bortolotto RP. Análise preliminar da relação entre emissão de $\mathrm{CO}_{2}$ do solo e do ecossistema em um agroecossistema do sul do Brasil. Ciência e Natura, 40: 251, 2018.

Veloso HP, Rangel-Filho ALR \& Lima JCA. Classificação da vegetação brasileira, adaptada a um sistema universal. Rio de Janeiro: IBGE, 123p, 1991.

Wilczak JM, Oncley SP \& Stage SA. Sonic anemometer tilt correction algorithms, Bound.-Lay. Meteorol., 99: 127-150, 2001.

Biodiversidade Brasileira - BioBrasil.

Edição Temática: Análise de Componentes do Sistema Climático e a Biodiversidade no Brasil

$$
\text { n. } 4,2021
$$

http://www.icmbio.gov.br/revistaeletronica/index.php/BioBR

Biodiversidade Brasileira é uma publicação eletrônica científica do Instituto Chico Mendes de

Conservação da Biodiversidade (ICMBio) que tem como objetivo fomentar a discussão e a disseminação de experiências em conservação e manejo, com foco em unidades de conservação e espécies ameaçadas.

ISSN: 2236-2886 